\title{
AROMA (AGU Robotic Optical Monitor for Astrophysical Objects)
}

\author{
Ichiro Takahashi, ${ }^{1}$ Kosuke Tsunashima, ${ }^{1}$ Takayuki Shiraki, ${ }^{1}$ Toru Kojima, ${ }^{1}$ \\ Atsumasa Yoshida, ${ }^{1}$ and Yujin E. Nakagawa ${ }^{2}$ \\ ${ }^{1}$ Department of Physics and Mathematics, Aoyama Gakuin University, 5-10-1 Fuchinobe, Sagamihara,
Kanagawa 229-8558, Japan
${ }^{2}$ Institute of Physical and Chemical Research, 2-1 Hirosawa, Wako, Sait ama 351-0198, Japan \\ Correspondence should be addressed to Ichiro Takahashi, itakahashi@phys.aoyama.ac.jp
}

Received 30 June 2009; Accepted 23 October 2009

Academic Editor: Taro Kotani

Copyright (C) 2010 Ichiro Takahashi et al. This is an open access article distributed under the Creative Commons Attribution License, which permits unrestricted use, distribution, and reproduction in any medium, provided the original work is properly cited.

\begin{abstract}
We are developing and operating the automatic telescope Aoyama Gakuin University Robotic Optical Monitor for Astrophysical objects (AROMA) in the Sagamihara Campus of Aoyama Gakuin University. AROMA is composed of two observational equipments, AROMA-N and AROMA-W. AROMA-N is a 30-cm aperture telescope system for rapid follow-up observations of the optical afterglow of gamma-ray bursts. Automatic analysis pipeline for AROMA-N has been developed and is working. The other observational equipment AROMA-W is a wide-field observation system using multiple digital single-lens reflex cameras. It covers a large FOV of about $35^{\circ} \times 25^{\circ}$. AROMAW provides photometric data in four bands with a limiting $\mathrm{V}$ magnitude of about 12-13 mag. Software to analyze massive image data automatically is being developed. We plan to monitor the luminosities of all the stars in the FOV of AROMA-W, detect optical transients, and trigger a follow-up observation with AROMA-N. We report on the development status and observational results of AROMA.
\end{abstract}

\section{Introduction}

There are various Optical Transients (OTs) which can be observed from ground based telescopes. Supernovae and novae are surveyed by various telescopes, and have increased the number of discoveries every year. Some Gamma-Ray Bursts (GRBs) are bright in optical [1]. In GRB 080319B, the brightest prompt optical emission that peaked at a visual magnitude of 5.3 was discovered during the burst [2]. Certain X-ray transients also have been observed not only in $\mathrm{X}$-rays but also in optical. Microquasar V4641 Sgr can usually be observed with magnitude of 12 in optical. It lightened up to $m=9$ correlated with X-rays in 1999. After that, a smallscale outburst is observed almost every year $[3,4]$. The Tago event, a nearby gravitational microlensing event, displayed brightening up to $8 \mathrm{mag}$ [5]. Comets and meteors also contribute to OTs. As for these phenomena, we cannot easily predict when and where they occur. To observe them before the onset, it is necessary to monitor the sky with a wide field of view and a high observational efficiency. Today these wide field cameras, utilizing CCDs and the commercial camera lenses, designed for this purpose have been established and are already in operations (e.g., WIDGET [6], Pi of the sky [7], MASTAR-VWF-4 [8], and RAPTOR 16 [9]). While these observational equipments are expensive, we are developing a low cost wide-field observation device AROMA-W that uses digital single-lens reflex cameras (DSLRs).

\section{AROMA}

We are developing and operating Aoyama Gakuin University Robotic Optical Monitor for Astrophysical objects (AROMA). AROMA is set up in the Sagamihara Campus of Aoyama Gakuin University (Latitude $=35.566^{\circ}$ and Longitude $=$ $\left.139.403^{\circ}\right)$. It is composed of two kinds of remote observation systems, AROMA-N and AROMA-W. Figure 1 is photographs of two equipments of AROMA. 


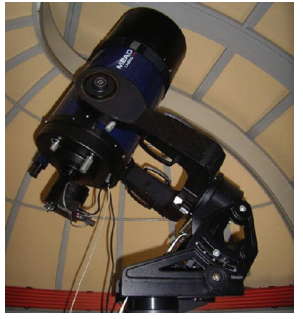

(a)

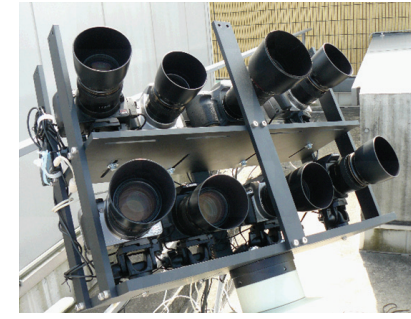

(b)
Figure 1: AROMA-N (a) and AROMA-W (b).

AROMA-N is a rapid follow-up telescope for astronomical transients. For GRB optical afterglows, this system autonomously makes rapid follow-up observations within tens of seconds based on positional information from satellites such as Swift. AROMA-N currently consists of a Schmidt-Cassegrain telescope with $30.5 \mathrm{~cm}$ aperture (MEADE LX200GPS-30), a Peltier cooled CCD camera (SBIG ST9-XE), and multiple standard filters (Clear, B, V, $\mathrm{R}$ and $\mathrm{I}$ ). The limiting magnitude is typically about $17 \mathrm{mag}$ in the Clear-band (in $100 \mathrm{~s}$ at $3 \sigma$ level). Automatic analysis pipeline is being developed in order to search for optical afterglows.

AROMA-W monitors the sky with a wide field of view. It has been developed since 2005. We have assembled the device and are starting initial operation now. The performance and the development of AROMA-W are described in the following sections.

\section{AROMA-W}

3.1. Instrumentation. AROMA-W consists of multiple DSLRs on an equatorial mount. The cameras used are one Canon EOS 5D with a EF200mm F2.8 USM and seven EOS 350Ds (EOS D Rebels) with EF100mm F2.0 USMs. Because their prices are about 1/10 compared with CCD cameras, a lot of those reserves can be prepared. As a result, they can easily exchange without a loss of the observation time even when they suffered from troubles in system such as a shutter failure. These cameras are set up in a purpose-built mount and put on equatorial mount "EM-400 Temma2". The cameras and the equatorial mount are operated remotely. The shutters of the cameras are controlled by the pulse from the DIO (Digital I/O) board in a Personal Computer (PC) so that the exposure of each camera is synchronized. The acquired data are transferred to PCs by USB every one frame. Since it takes time if the data of all cameras are transferred to one PC at a time, they are allotted with three PCs. The equatorial mount is controlled by the PC and will follow the field of view of the GRB observation satellites (e.g., Swift, Fermi). Figure 2 shows the schematic of AROMA-W system.

3.2. Multiple Bands Observation. The optical filters are placed on the image sensor of a DSLR along with a Bayer arrangement which has an alternating arrangement of three primary color red, green, and blue (here we call $\mathrm{R}^{\prime}, \mathrm{G}^{\prime}$

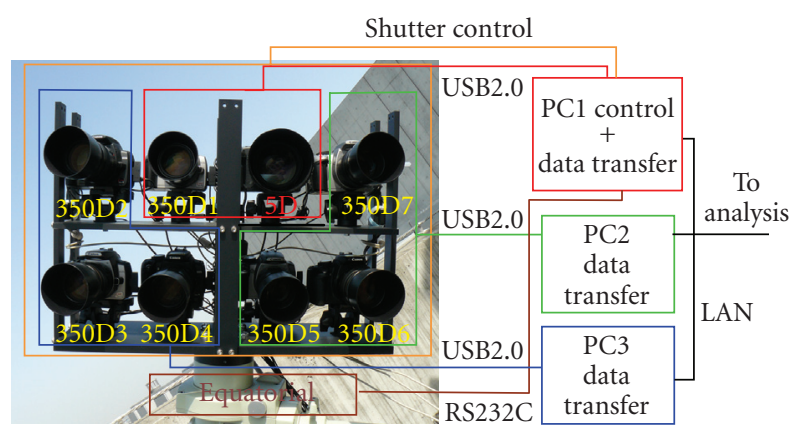

Figure 2: The schematic of AROMA-W system. Each camera and the equatorial mount are controlled by PC1. The observational data are acquired individually by the three PCs and forwarded to an analysis machine through LAN.

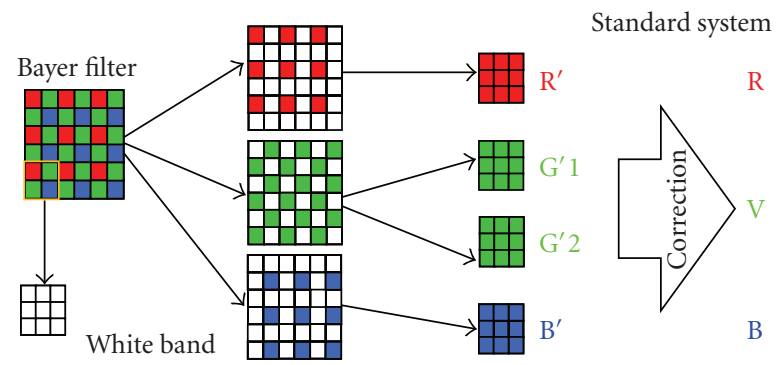

Figure 3: The schematic of the Tri-color imaging. Red, two, green, and blue filters are placed along with a Bayer arrangement on the image sensor. The $\mathrm{W}$ band image is made by adding the four images.

and $\mathrm{B}^{\prime}$ ) [10]. Simultaneous multiple-band observations are achieved by reading the data of these filters individually (Tricolor imaging). $\mathrm{W}$ band data which represent brightness information is obtained by adding the three-color data. Since the digital cameras employ $R^{\prime}, G^{\prime}$ and $B^{\prime}$ filters which differ a little from the standard system $(\mathrm{R}, \mathrm{V}$, and $\mathrm{B})$, the relation between our filter system and the standard system has been investigated by observing the same objects with various magnitude ranges simultaneously by both well-calibrated AROMA-N and AROMA-W. Then, a relative photometry between the filters was performed and magnitude relations (i.e., R-R', $V-\mathrm{G}^{\prime}$, and $\mathrm{B}-\mathrm{B}^{\prime}$ ) are obtained. For example, the relations for EOS $5 \mathrm{D}$ are well fitted by a linear function; $\mathrm{R}=$ $(0.88 \pm 0.02) \mathrm{R}^{\prime}-(3.1 \pm 0.3), \mathrm{B}=(0.93 \pm 0.02) \mathrm{B}^{\prime}-(0.2 \pm 0.3)$ and $\mathrm{V}=(0.90 \pm 0.01) \mathrm{G}^{\prime}-(2.8 \pm 0.2)$, where the quoted errors are $68.3 \%$ confidence levels. 350D also has a similar linearity. The standard system photometry with DSLRs can be done by correcting it according to this linearity. Figure 3 shows the schematic of the Tri-color imaging.

3.3. Field of View. The large field of view (FOV) of AROMA$\mathrm{W}$ consists of that of the multiple cameras to make a mosaic image. An EOS 5D with an EF200mm F2.8 USM has a FOV of $10.2^{\circ} \times 6.8^{\circ}$, and a 350D with an EF200mm F2.8 USM has that of $13^{\circ} \times 9^{\circ}$. A wide FOV of $35^{\circ} \times 25^{\circ}$ is covered with eight 


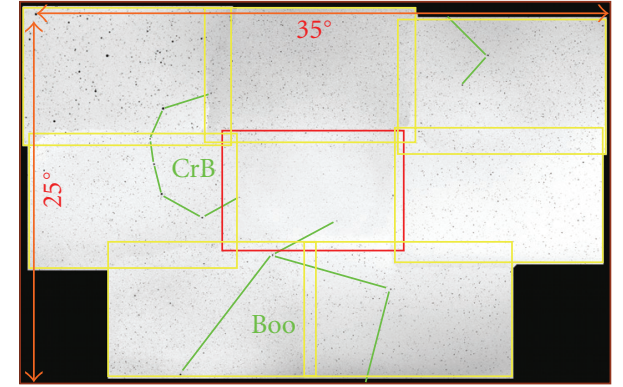

FIgure 4: The AROMA-W FOV. This is a combined image of 20 frames of $\mathrm{W}$ band exposed for $30 \mathrm{~s}$. The mosaic image shows the data taken by all eight cameras, in which the red rectangle displays the FOV of 5D and the yellow FOVs of 350Ds.

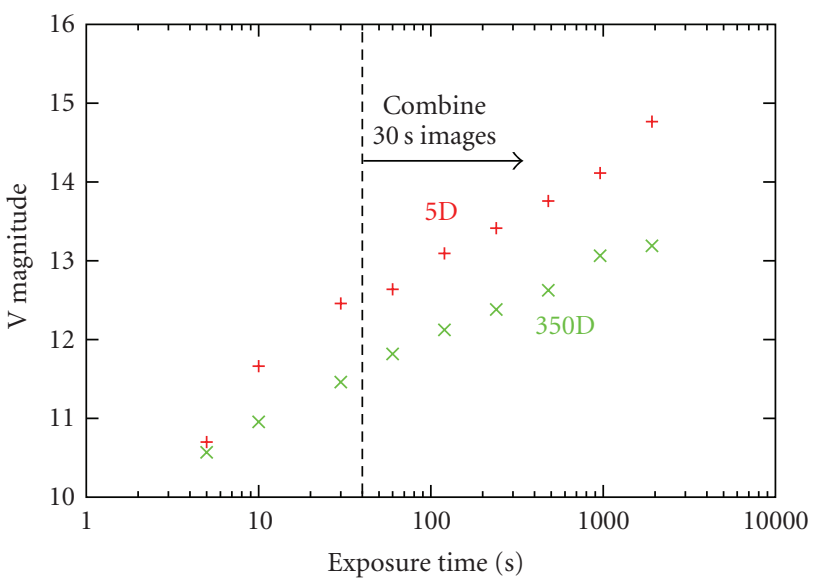

FIgURE 5: The summary of $\mathrm{W}$ band limiting magnitude with 5D and 350D. In the right side of the dashed line, the exposure time becomes long by combining the frames. There is a difference of about one magnitude between 5D and 350D.

cameras at a time. Figure 4 shows an image of the AROMA$\mathrm{W}$ FOV. The view of the $5 \mathrm{D}$ is arranged at the center, and the views of $350 \mathrm{D}$ are arranged in the surroundings.

3.4. Limiting Magnitude. The limiting magnitudes of each filter were investigated for all digital cameras. The AROMA site is near Tokyo, and the sky background condition is not so good. The $3 \sigma$ limiting magnitudes of EOS 5D $(20 \mathrm{~s}$ exposure, winter data) are $\mathrm{R}=12.1, \mathrm{~V}=12.8$ and $\mathrm{B}=13.5$. Those of $350 \mathrm{D}$ are $\mathrm{R}=11.9, \mathrm{~V}=12.3$, and $\mathrm{B}=13.4$. Figure 5 shows the summary of $\mathrm{W}$ band limiting magnitude with 5D and 350D. This implies that our new system can detect GRB optical flashes like GRB 990123 with $\sim 9$ magnitude and GRB 080319B with $\sim 5.3$ magnitude. It is known that supernovae can be very bright in optical and X-ray band. Since the time scale of their decay is tens of days, and the optical peak is about 12 13 mag (V band), AROMA-W can detect such a peak with a few minutes exposure. Some X-ray novae also can be detected with the sensitivity of AROMAW. Generally, after an X-ray nova is detected with X-ray detectors, observations in the optical band are conducted.
If an extensively large FOV monitor observation, such as those possibly by AROMA-W, is running during almost all the night, a detection in optical could be made in advance of X-rays. For example, V4641 Sgr becomes bright almost every year and it became as bright as 9 mag. in September, 1999. Moreover, sensitivity limits of AROMA-W are also appropriate to detect the large amplitude flares of BL-LACs and OVV (optically violent variable) quasars.

\section{Real-Time Analysis of AROMA-W}

We are developing the real-time analysis pipeline for AROMA-W. The data will be analyzed automatically in parallel with observations. Basic processes of the pipeline are as follows. First, the data of AROMA-W are transmitted from Compact Flash (CF) in the DSLRs to the analysis PCs via the transfer PCs. The dead time between frames can be shortened to about 3 seconds by transferring data while a following frame is exposed. When data are transmitted to the analysis PCs, the image processing is started. The RAW data are converted to FITS, and then the data reduction is processed; subtracting dark noise and correction of flat pattern, after those, resolving data into tri-colors and positional corrections are performed. Next, the main analysis starts; detection of astronomical objects and comparison with star catalogs (mainly USNO B1.0) follow. About 2000 to 5000 objects are detectable from the data of each camera. By comparing photometry data of these stars with the previous frame data, brightening OTs and fading stars are searched. Light curves of magnitude and flux ratio are obtained for all the objects within the AROMA FOV by the differential photometry. The variability of each light curve is investigated at certain interval, and if it exceeds a certain threshold, it is recorded and/or triggers an alert automatically. The development of the pipeline has progressed to produce light curves until now. The rejection algorithm of the false events will be added to the current software in the future. By monitoring light curves continuously, variable stars and transient objects (GRB, SNe, X-ray novae, etc.) will become detectable. Furthermore, an unexpected brightening and variability may also be detectable.

\section{Test Observation}

We have tested the performance to detect variable stars several times so far by using the analytical pipeline described in the previous section. Figure 6 shows examples of variable stars' light curves which were detected by the real-time analysis. Various variable stars are detected automatically during the test, though the observation time is short. However, there is still a problem in the detection program. Tracks of airplanes and double star, and so forth are misidentified as OTs.

\section{Conclusions}

In order to observe OTs of GRBs, SNe, X-Ray novae, variable stars, and so forth, we are developing AROMA-W: 


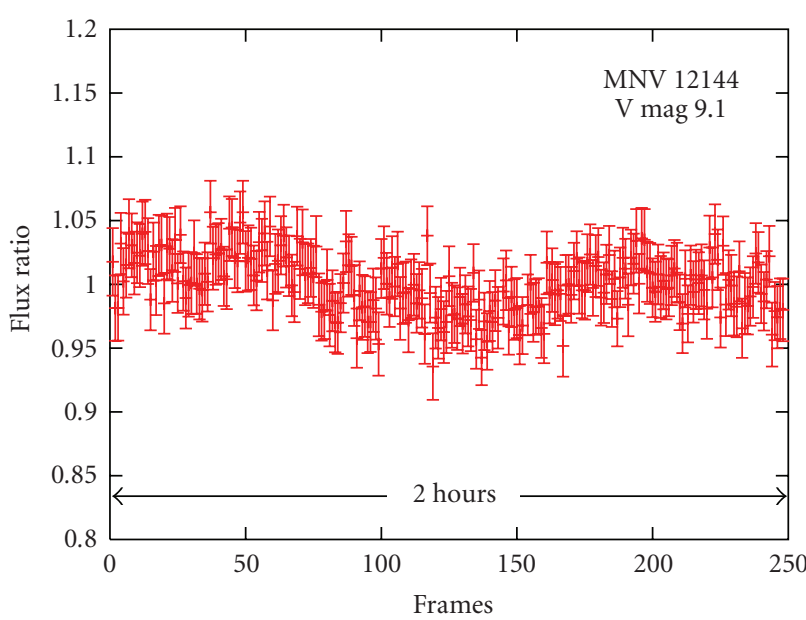

(a)

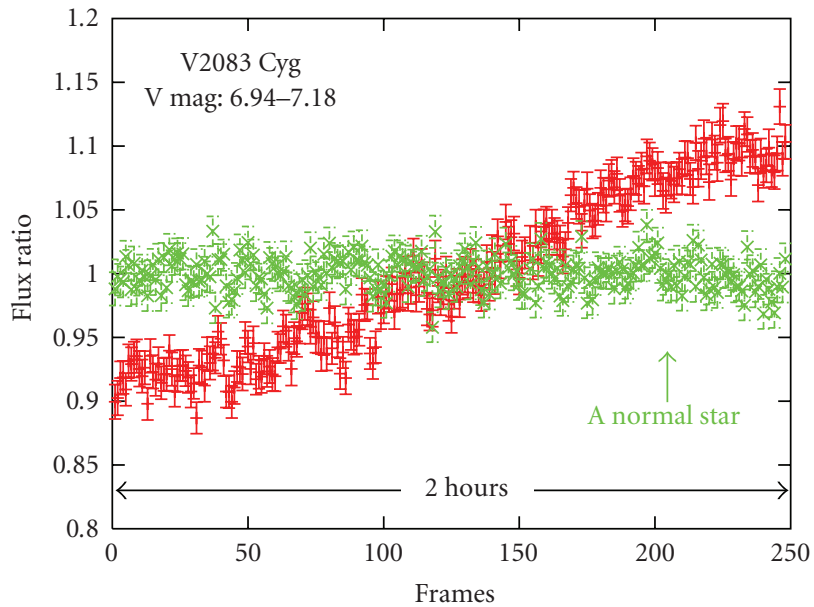

(c)

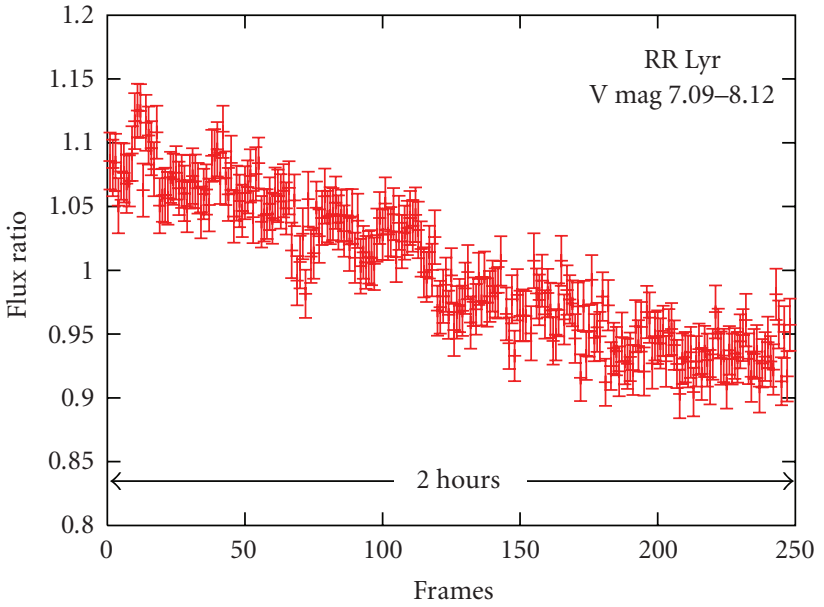

(b)

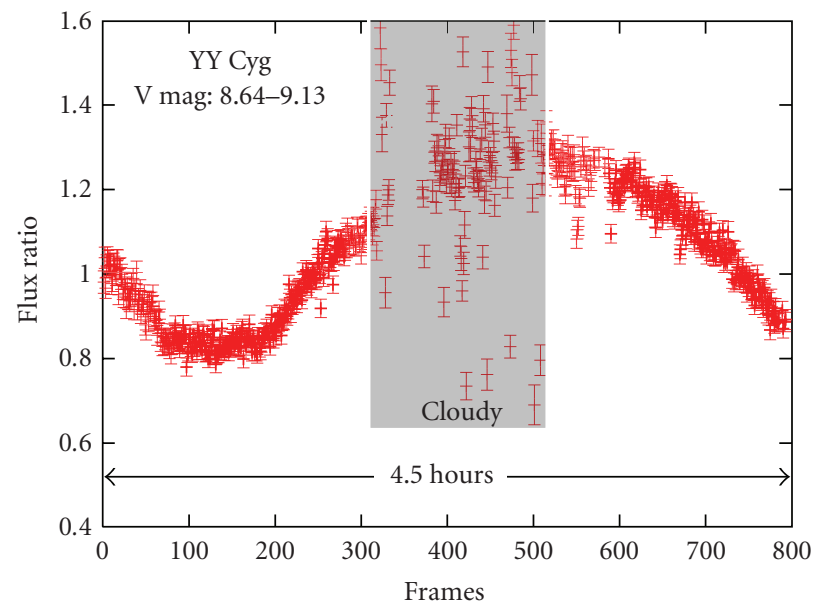

(d)

FIGURE 6: Examples of variable star's light curves which were detected by real-time analysis. In the panel of V2083, the light curve of a nearby usual star is plotted in green. Unfortunately, it became cloudy partly during the observation of YY Cyg; this part is colored in gray.

the full-time wide-field observation system using multiple digital single-lens reflex cameras. AROMA-W can detect stars with brightness down to $12-13$ mag in the field of view of $35^{\circ} \times 25^{\circ}$ with an exposure time of $20 \mathrm{~s}$. We are currently developing a real-time analysis pipeline, which will work in parallel with the observation. It detects OTs and makes the light curves. The test observation by AROMA-W was done, and some already-known variable stars have been detected automatically.

The next steps we foresee are the followings:

(i) starting main observations of AROMA-W,

(ii) improving analytical pipeline,

(iii) building the data archive,

(iv) setting up the AROMA website,

(v) coordinating observation of AROMA-W and AROMA-N, (vi) aiming at real-time detection of OTs by AROMA$\mathrm{W}$ to reporting it promptly to various telescopes worldwide.

\section{References}

[1] J. Van Paradijs, P. J. Groot, T. Galama, et al., “Transient optical emission from the error box of the $\gamma$-ray burst of 28 February 1997," Nature, vol. 386, pp. 686-689, 1997.

[2] J. L. Racusin, S. V. Karpov, M. Sokolowski, et al., "Broadband observations of the naked-eye $\gamma$-ray burst," Nature, vol. 455, pp. 183-188, 2008.

[3] M. Uemura, R. E. Mennickent, and R. Ishioka, "Rapid optical fluctuations in the black hole binary V4641 sagittarii," Publications of the Astronomical Society of Japan, vol. 54, pp. 95-101, 2002.

[4] M. Uemura, et al., "Optical observation of the 2003 outburst of a black hole X-ray binary, V4641 Sagittarii," Publications of the Astronomical Society of Japan, vol. 56, pp. 823-829, 2004.

[5] A. Fukui, F. Abe, K. Ayani, et al., "Observation of the first gravitational microlensing event in a sparse stellar field: the 
tago event," The Astrophysical Journal, vol. 670, pp. 423-427, 2007.

[6] T. Tamagawa, K. Torii, M. Matsuoka, et al., "Spectral analysis of 35 GRBs/XRFs observed with HETE-2/FREGATE," New Community of Ichthus Mission Church, vol. 28, pp. 771-774, 2005.

[7] M. Sokolowski, et al., "Investigation of astrophysical phenomena in short time scales with "Pi of the Sky" apparatus," http://grb.fuw.edu.pl/.

[8] http://apollo.sai.msu.ru/.

[9] http://www.raptor.lanl.gov/.

[10] B. E. Bayer, US patent no. 3,971,065, July 1976. 

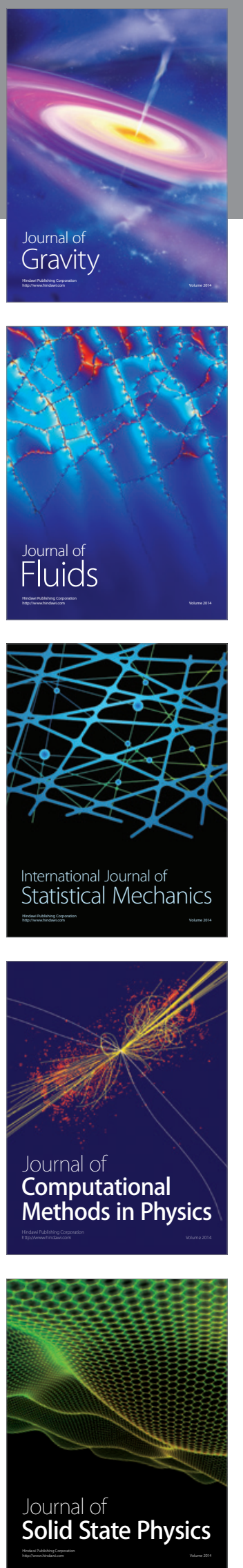

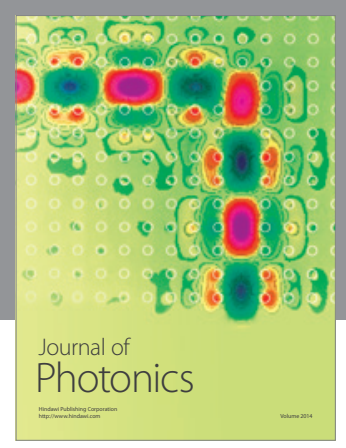

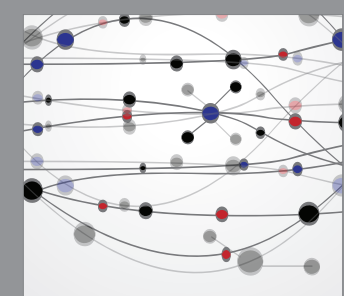

The Scientific World Journal
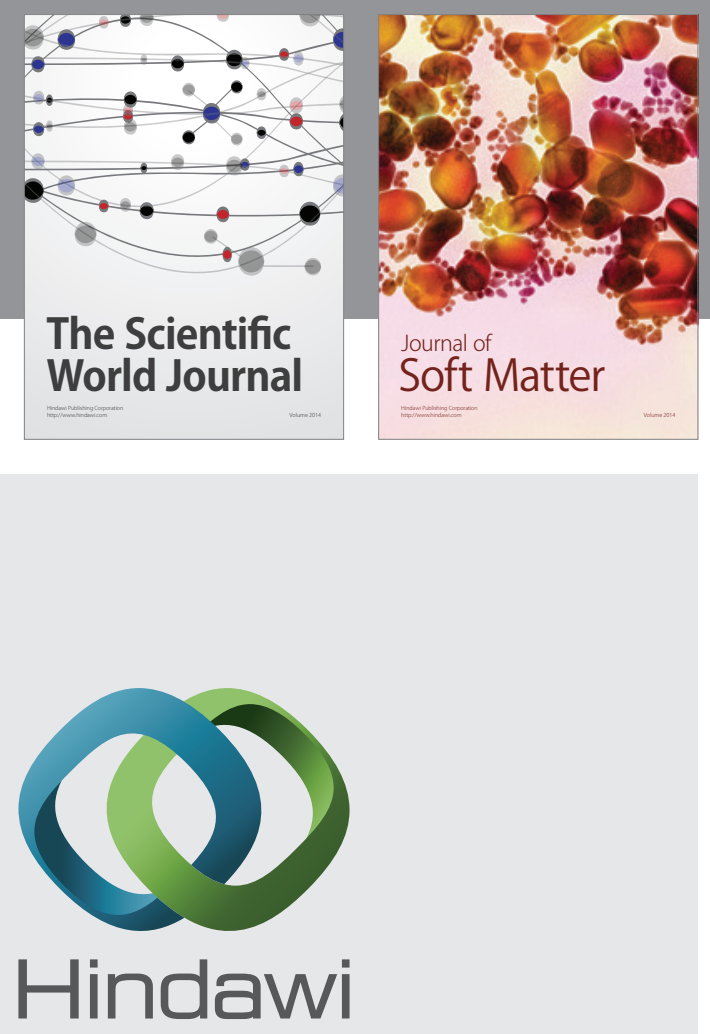

Submit your manuscripts at

http://www.hindawi.com
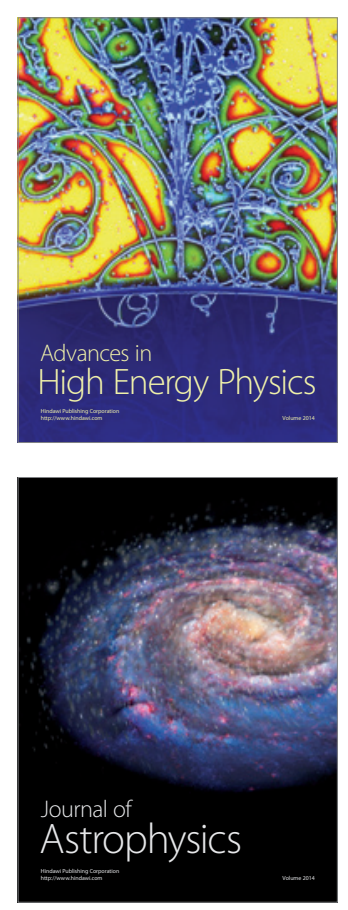
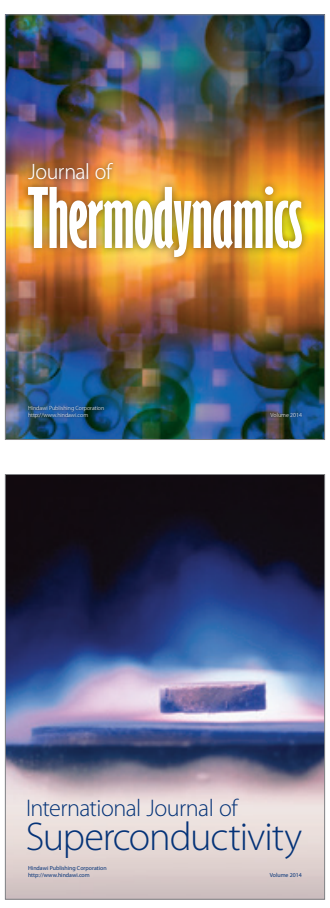
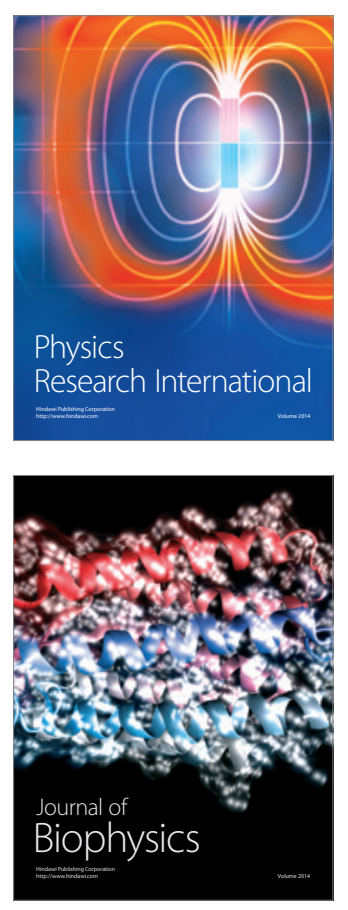
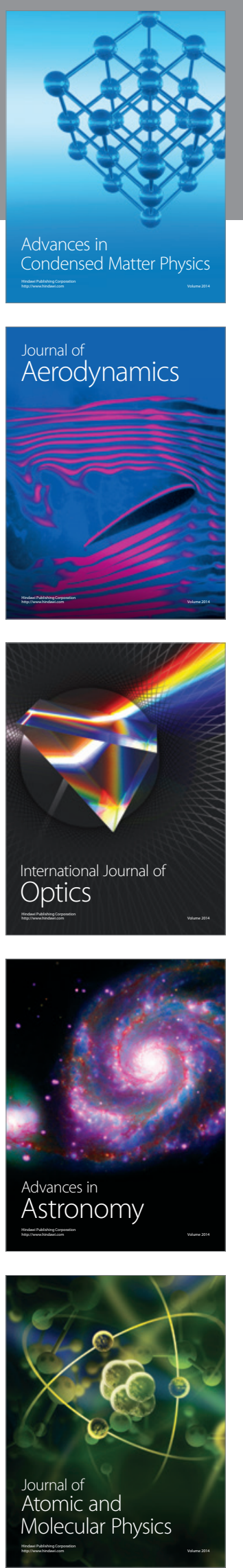